\title{
ARTICLE
}

\section{Acidic ambiance induced post-oxidative stress affects AMPK- PGC1a-SIRT1 axis in the skeletal muscles of zebrafish Danio rerio Hamilton, 1822}

\author{
Sabarna Chowdhury', Subhendu K Chatterjee ${ }^{1}$, Samir Bhattacharya ${ }^{2}$, Sudipta Maitra ${ }^{3}$ \\ and Surjya K Saikia ${ }^{1 *}$ \\ ${ }^{1}$ Aquatic Ecology and Fish Biology Laboratory, Department of Zoology, Centre for Advanced Studies, Visva-Bharati \\ (A Central University), Santiniketan, West Bengal, India \\ ${ }^{2}$ Molecular Endocrinology Laboratory, Department of Zoology, Centre for Advanced Studies, Visva-Bharati (A Central \\ University), Santiniketan, West Bengal, India / Currently at The University of Burdwan, Golapbag, Barddhaman, \\ West Bengal, India \\ ${ }^{3}$ Molecular and Cellular Endocrinology Laboratory, Department of Zoology, Centre for Advanced Studies, Visva- \\ Bharati (A Central University), Santiniketan, West Bengal, India
}

\begin{abstract}
The present study was aimed to understand whether acidic $\mathrm{pH}$ induces oxidative stress in zebrafish affecting metabolic sensor protein and thereby, the mitochondrial functions in the skeletal muscle of zebrafish. The experiments performed in aquaria involved the study of the changes of HIF1a, AMPK, PGC1a and SIRT1 levels together with the levels of mitochondrial Tfam and Nrf1. The results obtained from investigation of superoxide dismutase (SOD), catalase and glutathione revealed that the fish undergoes oxidative stress within a short duration of exposure to acidic ambiance. Further analysis with MDA and HIF1a helped to understand the effects of post oxidative stress on skeletal muscle of the fish at pH $5.0( \pm 0.5)$. Of the three tissues studied (gill, brain and skeletal muscle) the effect was maximum in skeletal muscle as depicted by MDA level at 2 hours beyond which it declines augmenting death or mortality (15\%) to the fish. Consequently, HIF1 a was increased as an adaptive strategy against metabolic disruption during the first 2 hours period. However, on exposure to $\mathrm{pH} 5.0( \pm 0.5)$ for 2 hours, there were decrease of the metabolic sensors viz. AMPK and SIRT1 followed by mitochondrial gene transcriptional co-activator PGC1a. The expression of mitochondrial transcription factors Tfam and Nrf1 were also reduced confirming perturbation in mitochondrial function affecting low ATP production compared to control. This was also supported by the decrease of COXII as well as mitochondrial complex I activity. All these results confirm that the metabolic machinery of zebrafish is affected when $\mathrm{pH}$ was lowered to $5.0( \pm 0.5)$.

Acta Biol Szeged 64(2):191-198 (2020)
\end{abstract}

\section{KEY WORDS}

AMPK

malondialdehyde mitochondrial bioenergetics mitochondrial biogenesis PGC1a

SIRT1

\section{ARTICLE INFORMATION}

Submitted

12 November 2020

Accepted

28 December 2020

*Corresponding author

E-mail: surjyasurjya@gmail.com

\section{INTRODUCTION}

For last forty years, acidification in water bodies, largely in lakes throughout the world has invited extensive and vigorous research approach regarding aquatic resource management (Mcdonald 1983). Acidification of freshwater bodies became a global problem involving Europe, USA and Asia (Schindler 1988; Psenner 1994). Aquatic animals including fish live in a narrow range of $\mathrm{pH}$, which is near neutral, therefore, any change in $\mathrm{pH}$ range adversely affects their normal biological functioning (Mcdonald 1983; Wood 1989). Of these, the metabolic mechanisms are found to be the most susceptible biological functions (Bhaskar and Govindappa 1985). A high alkaline or acidic stress may affect the physiological system, especially ion transporting cells in fish (Kwong et al. 2014). Thus, a sudden change in $\mathrm{pH}$ may lead to acute stress and a chronic $\mathrm{pH}$ state may cause death. Besides, acidic environment induced physiological impairments may disturb the overall homeostatic condition during growth in fish.

The zebrafish (Danio rerio), a small teleost has become the most widely used animal model for the study of various biological phenomena of vertebrates. Recently, this fish was subjected to study under different stress conditions for stress related diseases (Steenberger et al. 2011). However, very limited studies on the stress tolerance, especially with $\mathrm{pH}$ are performed on zebrafish. Reported studies address toxicological effect on embryos of zebrafish (Dave 1984, 1985; Andrade et al. 2017). Zahangir et al. 
(2015) reported secondary stress responses of ' $\mathrm{pH}$ stress' on zebrafish where the haematological parameters were primarily assessed. However, metabolic regulator proteins related to mitochondrial functions due to $\mathrm{pH}$ stress have not been studied, yet.

In the present study, responses during oxidative stress and its post traumatic consequences caused by acidic $\mathrm{pH}$ were analysed through oxidative stress indicators and specified biochemical parameters, especially malondialdehyde (MDA) level in fish body. Being a by-product of lipid peroxidation, MDA has been widely used as a biochemical index of oxidative stress in mammals (Eze et al. 2008). The results of MDA, therefore, supported the changes of oxidative stress indicators like superoxide dismutase (SOD) and catalase enzyme activity and glutathione. The hypoxia inducible factor- $1 \alpha$ (HIF1 $\alpha$ ) from skeletal muscle tissue was also studied to affirm the oxidative stress. At first instance, analysis of metabolic regulator proteins like AMP-activated protein kinase (AMPK), peroxisome proliferator-activated receptor gamma coactivator- $1 \alpha$ (PGC1 $\alpha$ ) and sirtuin 1 (SIRT1 or NAD-dependent deacetylase sirtuin-1) were performed, which was followed by the analysis of transcription factor A of mitochondria (Tfam) and nuclear respiratory factor 1 (Nrf1). These were followed by the analysis of mitochondrial complex I activity, expression of cytochrome oxidase II (COX II) and synthesis of ATP to elucidate a better understanding on the metabolic impairments.

\section{MATERIALS AND METHODS}

\section{Stress exposure to zebrafish}

Zebrafish stock was kept in aquarium under laboratory environment ( $\mathrm{pH} 6.5-7.5$, temperature $25-28^{\circ} \mathrm{C}$, dissolved oxygen (DO) $7-10 \mathrm{mg} / \mathrm{l}$ ). After one week of acclimatization, zebrafish (weight: $0.7 \mathrm{~g}$, total length: $3.8 \mathrm{~cm}, \mathrm{n}=10$ ) were collected in separate aquarium for further study. The experiments were set with three different levels of $\mathrm{pH}$ to measure the responses of tissues against acidic stress. These three $\mathrm{pH}$ levels were acidic (AC, $5.0 \pm 0.5$ ), near neutral (NN, $6.0 \pm 0.45)$ and neutral $(\mathrm{N}, 7.0 \pm 0.3)$. The $\mathrm{pH}$ levels were maintained by application of weak organic acid following a regression equation as $\mathrm{Y}=7.675-0.008 \mathrm{X}$ $\left(\mathrm{Y}=\mathrm{pH}\right.$ and $\mathrm{X}=$ Volume acetic acid, $\left.\mu \mathrm{l}, \mathrm{R}^{2}=0.997\right)$. A portable digital pocket-sized $\mathrm{pH}$ meter (HI98107P) was used to record the $\mathrm{pH}$ of the aquaria. The responses in fish tissues were measured in terms of MDA level from the respective tissues. For each analysis, ten fishes were sampled for collection of tissues.

Three tissues (skeletal muscle, brain and gills) were evaluated for maximum stress response against the three $\mathrm{pH}$ levels, viz. AC, NN and $\mathrm{N}$. The values were recorded till the first mortality occurred in the treated fish population. Two norms were standardized after these three experiments. These were, (i) the highly responsive tissue and (ii) the effective $\mathrm{pH}$ level to assess the biochemical parameters of the highly responsive tissue. Once the above norms were set, a new set of fish were treated with the effective $\mathrm{pH}$ level and only the highly responsive tissue was subjected to all specified biochemical analysis. In this case, the effective duration of treatment period (mortality $\approx 15 \%$ ) was determined beforehand.

To understand the progression and resulting effect of acidic stress, the specified biochemical parameters like SOD, catalase, and glutathione levels were measured for a period of two hours (the effective duration at $15 \%$ mortality) with $30 \mathrm{~min}$ intervals. The tissue samples thus collected were also used for assessing levels of metabolic sensors related to mitochondria after $2 \mathrm{~h}$ of acidic stress exposure.

Although not mandatory for the purpose, attempts were made to maintain all ethical norms for the fish in the subject during the experiment. Before tissue collection, fishes were narcotized using MS222.

\section{Tissue collection and processing}

Tissues (skeletal muscle, gill and brain) were collected (n $=10$ ) from zebrafish and processed for further analysis. Before every analysis, the pooled tissues kept in lysis buffer (phosphate buffer) were homogenized using micro tissue homogenizer. The tissues homogenized were then centrifuged in $10000 \mathrm{~g}$ for $15 \mathrm{~min}$. The supernatant was collected and used for all biochemical assessment. For western blot analysis, tissue proteins of $100 \mu \mathrm{g}$ from the lysate were used.

\section{Biochemical assays}

The biochemical assessment was performed for MDA, other antioxidant enzymes and antioxidant (SOD, catalase, glutathione), mitochondrial complex I and ATP. MDA assay was performed according to the method of Aust (1985). MDA is a product of lipid peroxidation and reacts with TBA (thiobarbituric acid) to give a red species named TBARS (thiobarbituric acid reactive substance). The antioxidant enzyme SOD assay was performed following the method of Ewing and Janero (1995), while catalase assay together with reduced glutathione quantification were performed using microplate assay kits (G-Biosciences, ITAK1061 and ITAK1006). The ATP produced and the mitochondrial complex I activity were quantified using microplate assay kits (ATP assay kit, Sigma MAK190 and Cayman Chemical Mitocheck, Mitochondrial Complex I activity assay kit 700930). 


\section{Electrophoresis and immunoblotting}

The desired quantity of tissue protein was resolved on 10\% SDS-PAGE and were transferred to PVDF membranes through transfer buffer $(25 \mathrm{mM}$ Tris, $193 \mathrm{mM}$ glycine, 20\% methanol, $\mathrm{pH} 8.5$ ) for 1.5 hours. Western blot analysis was performed for HIF1 $\alpha$, PGC1 $\alpha$, AMPK, SIRT1, Tfam, Nrf1 and COXII using specific antibodies (Table 1) against the protein/factor of interest. Membrane bound primary antibodies were visualized using corresponding secondary antibodies at 1:1000 dilutions, which was tagged with alkaline phosphatase and developed with corresponding substrates, 5-bromo-3-chloro-3-indolyl phosphate/nitrobluetetrazolium (BCIP/NBT). Band intensities were quantified by utilizing Image J software (NIH, Bethesda, MD).

\section{Statistical analysis}

Homogeneity of variances of data sets were tested using Levene's statistics. The Kruskal-Wallis $\mathrm{H}$ test was computed where Levene's statistics did not comply to $\mathrm{p}$ $>0.05$. In case of all analyses, $\alpha$ level was fixed at 0.05 . SPSS 16.0 was used for all statistical analyses.

\section{RESULTS}

The present study examined three different tissues from adult zebrafish, which were subjected to three $\mathrm{pH}$ levels, viz., AC, NN and N (control). The formation of MDA in skeletal muscle tissue was significantly higher than the control (Kruskal-Wallis H Test, $\chi 2=25.806$, $\mathrm{p}=0.00$;

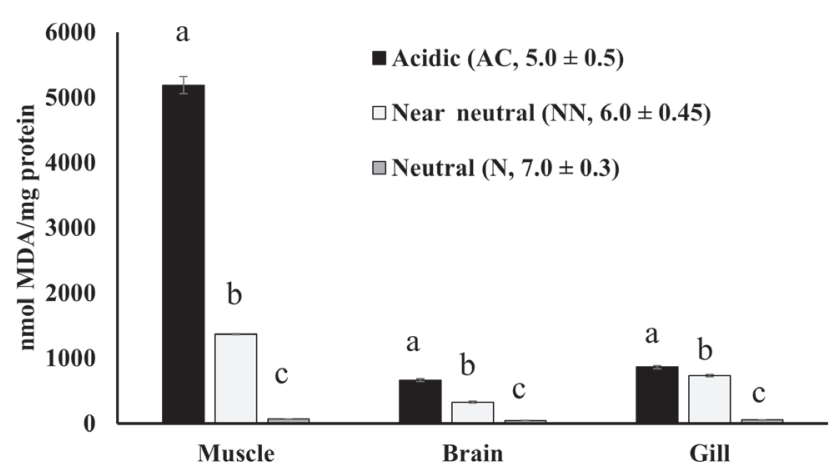

Figure 1. Determination of MDA levels in three different tissues (skeletal muscle, brain and gills) of zebrafish under three different $\mathrm{pH}$ conditions: acidic (AC, $5.0 \pm 0.5)$, near neutral (NN, $6.0 \pm 0.45)$ and neutral $(\mathrm{N}, 7.0 \pm 0.3)$. Data are shown as Mean $\pm \mathrm{SE}$. Bars with different lower-case letters show statistically significant difference at $p<0.05$.

Fig. 1). Out of all the three $\mathrm{pH}$ levels, mean rank of $\mathrm{pH}$ $5.0 \pm 0.5$ showed maximum effect on skeletal muscle as reflected from MDA levels (Fig. 1). It was also observed that increased formation of MDA in skeletal muscle tissue was comparatively higher than brain and gill tissues (Fig. 1). We have, therefore, considered skeletal muscle of zebrafish as primary tissue affected by acidic ambiance. The difference in the formation of MDA in skeletal muscle among three $\mathrm{pH}$ levels (namely AC, NN and $\mathrm{N}$ ), were found statistically significant (Kruskal-Wallis $\mathrm{H}$ Test, $\chi 2=25.806, p=0.00$; Fig. 1). Here, the AC showed highest rank in terms of MDA formation in skeletal muscle compared to other $\mathrm{pH}$ levels. The maximum level
(A)

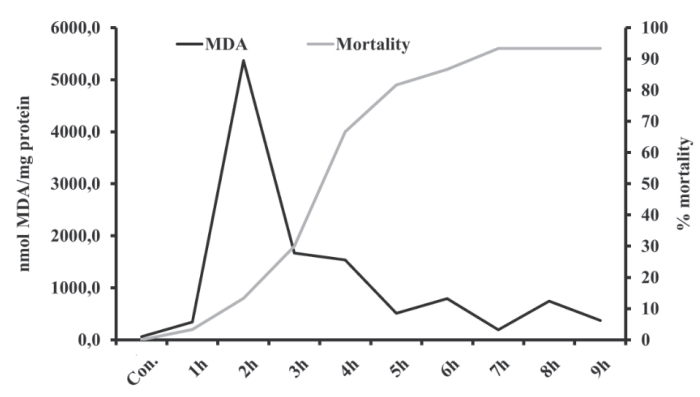

(B)

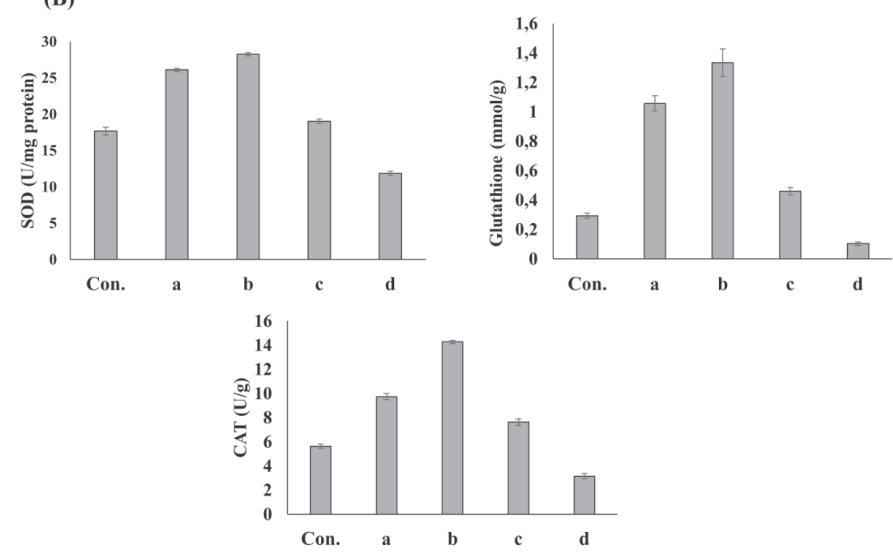

Figure 2. Formation of MDA in skeletal muscle tissue of zebrafish. (A) Formation of MDA in skeletal muscle tissue and percent mortality estimated over 9-h gradient with $1 \mathrm{~h}$ interval. The MDA formation was highest on exposure for $2 \mathrm{~h}$ to $\mathrm{pH} 5$ ( \pm 0.5 ). (B) catalase, glutathione and SOD produced under $\mathrm{pH} 5.0 \pm 0.5$ in the zebrafish skeletal muscle when treated for $30 \mathrm{~min}, 1 \mathrm{~h}, 1.5 \mathrm{~h}$ and $2 \mathrm{~h}$. Bars with different lower-case letters show statistically significant difference at $\mathrm{p}<0.05$.

On figure B: a, pH $5 \pm 0.5$ for 30 min; b, pH $5 \pm 0.5$ for 1 h; $\mathbf{c}, \mathrm{pH} 5 \pm 0.5$ for 1.5 h; d, $\mathrm{pH} 5 \pm 0.5$ for $2 \mathrm{~h}$ 

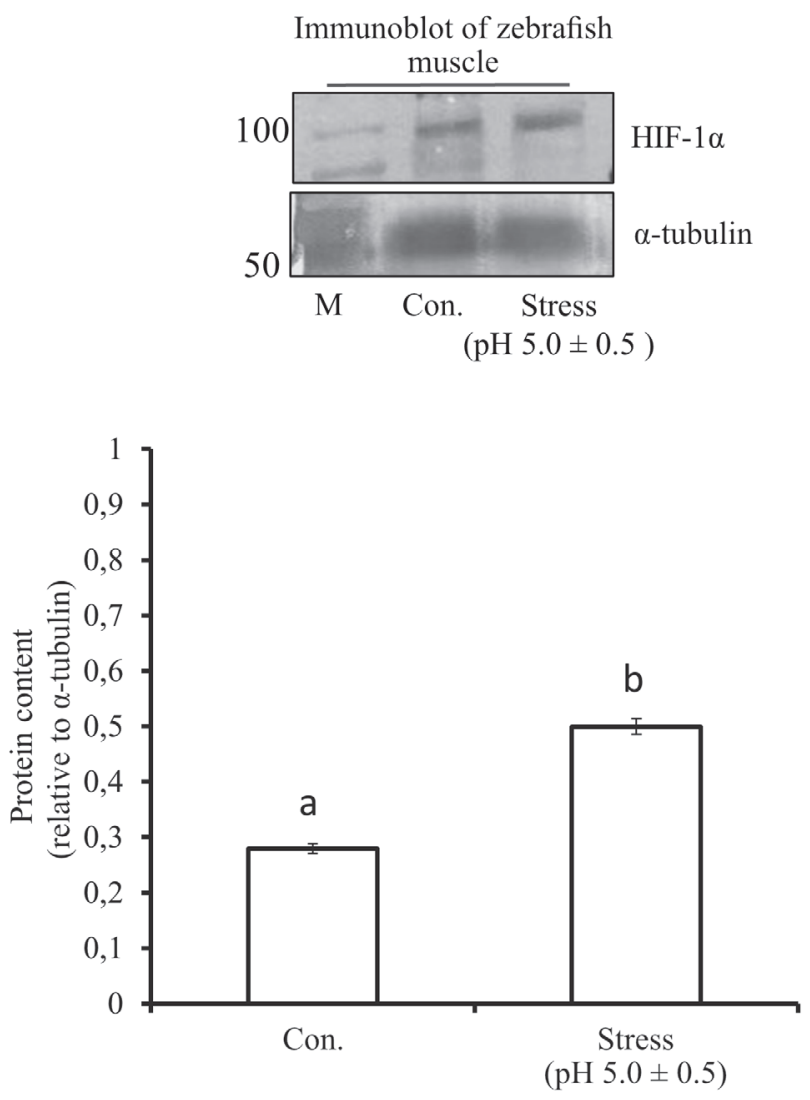

Figure 3. Immunoblot of a stress marker protein, HIF1a and its densitometry analysis after $2 \mathrm{~h}$ of stress exposure of zebrafish to $\mathrm{pH} 5.0$ \pm 0.5 . Data are shown as Mean $\pm \mathrm{SE}$. Bars with different lower-case letters show statistically significant difference at $p<0.05$.

of MDA in skeletal muscle tissue (5000 nmol MDA/mg protein) was recorded at the second hour of treatment. Beyond this period, fish mortality starts (Fig. 2A). Thus, it was standardized that the skeletal muscle is the highly responsive tissue for oxidative stress induced by acidic ambiance, and it experiences elevated oxidative stress during 2-hours-exposure to acidic ambiance. All these outcomes were supported by the results of SOD, catalase and glutathione assays of the skeletal muscle of fish at 30 min, $1 \mathrm{~h}, 1.5 \mathrm{~h}$ and $2 \mathrm{~h}$ intervals under $\mathrm{pH} 5.0 \pm 0.5$. The results showed that, compared to control, the acidic ambiance markedly induced the elevation of catalase and SOD antioxidant enzymes activities along with the oxidative stress protective glutathione compound between $30 \mathrm{~min}$ to $1 \mathrm{~h}$ of exposure to acidic stress. Beyond this period, at $2 \mathrm{~h}$, the activities of antioxidant enzymes (catalase and SOD) and glutathione were declined steeply and reached below the normal level (control) (Fig. 2B). The KruskalWallis $\mathrm{H}$ Test showed statistically significant difference of activities of $\operatorname{SOD}\left(\chi^{2}=45.155, \mathrm{p}=0.000\right)$, glutathione
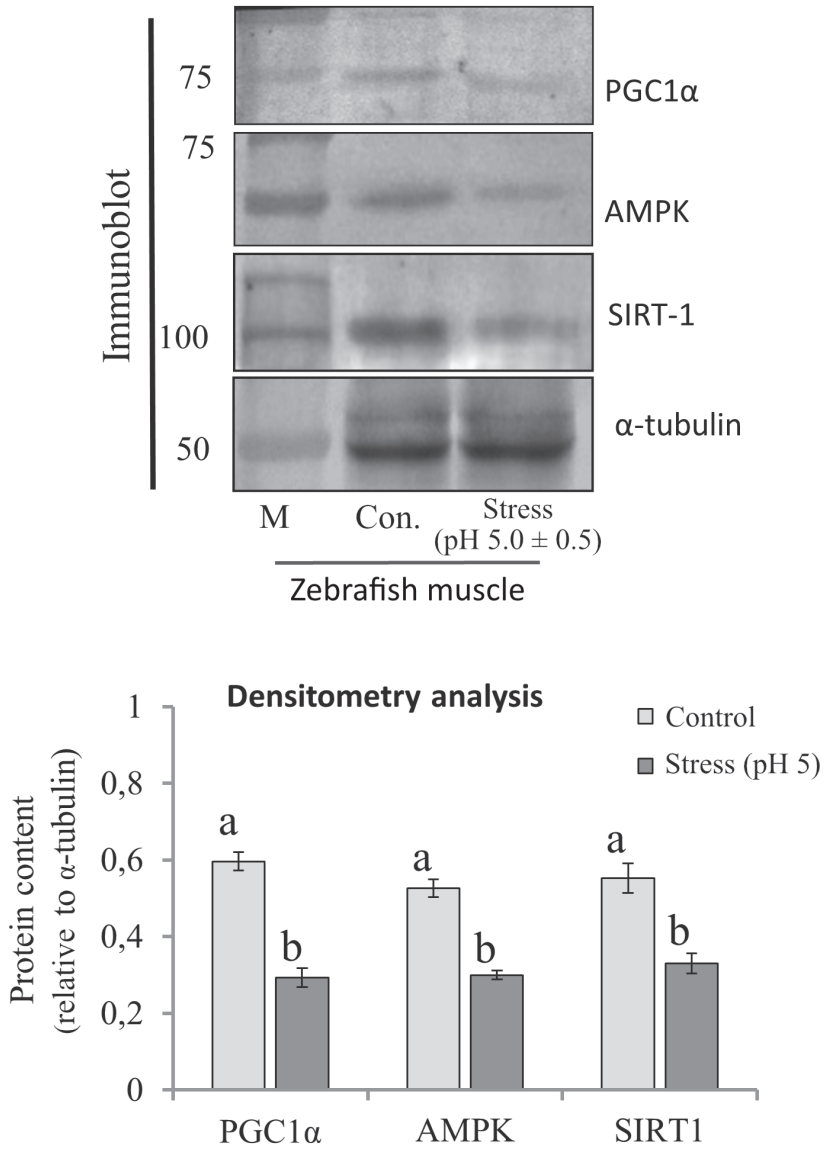

Figure 4. Immunoblots of AMPK, SIRT1 and PGC1 $a$ and, their densitometry analysis compared to control after $2 \mathrm{~h}$ of stress exposure of zebrafish to $\mathrm{pH} 5.0 \pm 0.5$. Data are shown as Mean $\pm \mathrm{SE}$. Bars with different lower-case letters show statistically significant difference at $p<0.05$.

$(\chi 2=45.161, \mathrm{p}=0.00)$ and catalase $\left(\chi^{2}=46.874, \mathrm{p}=0.00\right)$ from the control. HIF- $1 \alpha$, an oxidative stress marker protein was also significantly increased at pH $5.0 \pm 0.5$. There is a significant increase in the expression of the protein $\left(\chi^{2}=8.308, \mathrm{p}=0.004\right)$ than the control, which is quite prominent from the densitometric analysis, when exposed under acidic ambiance for $2 \mathrm{~h}$ (Fig. 3).

Immunoblots of AMPK, PGC1 $\alpha$ and SIRT1 from skeletal muscle tissue showed that the amount of AMPK, PGC1 $\alpha$ and SIRT1 were decreased under acidic pH (Fig. 4). Both the metabolic sensors (AMPK and SIRT1) were significantly decreased $\left(\chi 2=8.366, \mathrm{p}=0.004\right.$ and $\chi^{2}=$ $7.908, \mathrm{p}=0.005$, respectively), which in turn resulted in the significant decreased amount of the master regulator PGC1 $\alpha\left(\chi_{2}=8.396, \mathrm{p}=0.004\right)$, which is the transcriptional coactivator of the energy homeostatic pathway in mitochondria. It is reasonable to think that reduced level of PGC1 $\alpha$ results in the decrease in expressions of the PGC1 $\alpha$-regulated Tfam, Nrf1 (transcription factors in- 
(A)
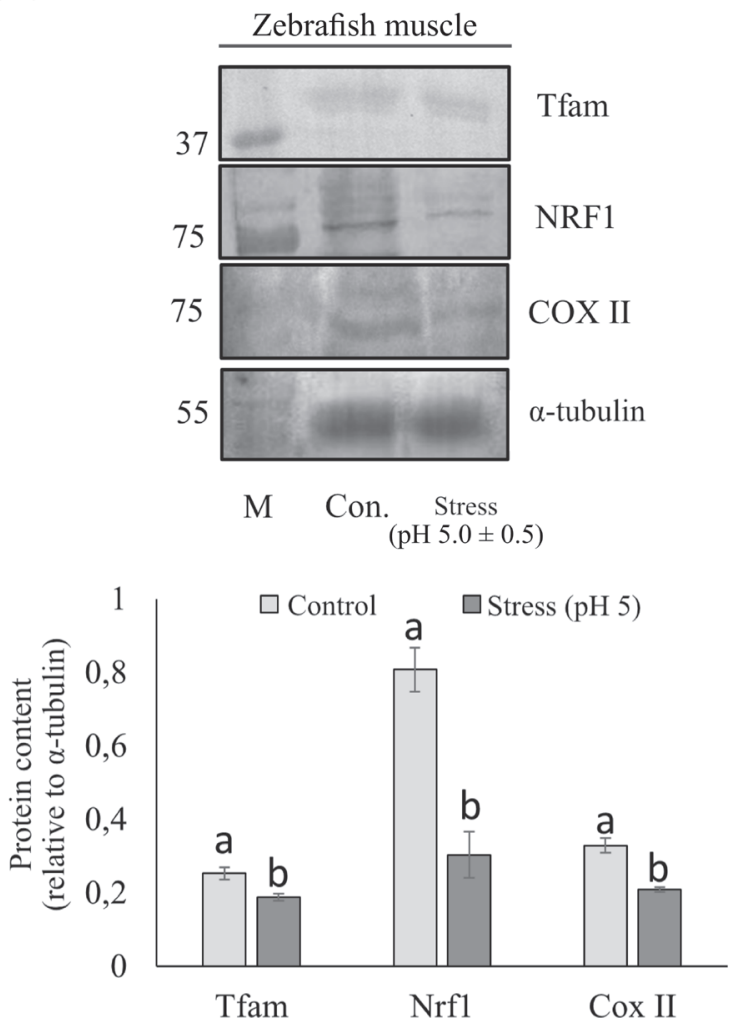

(B)
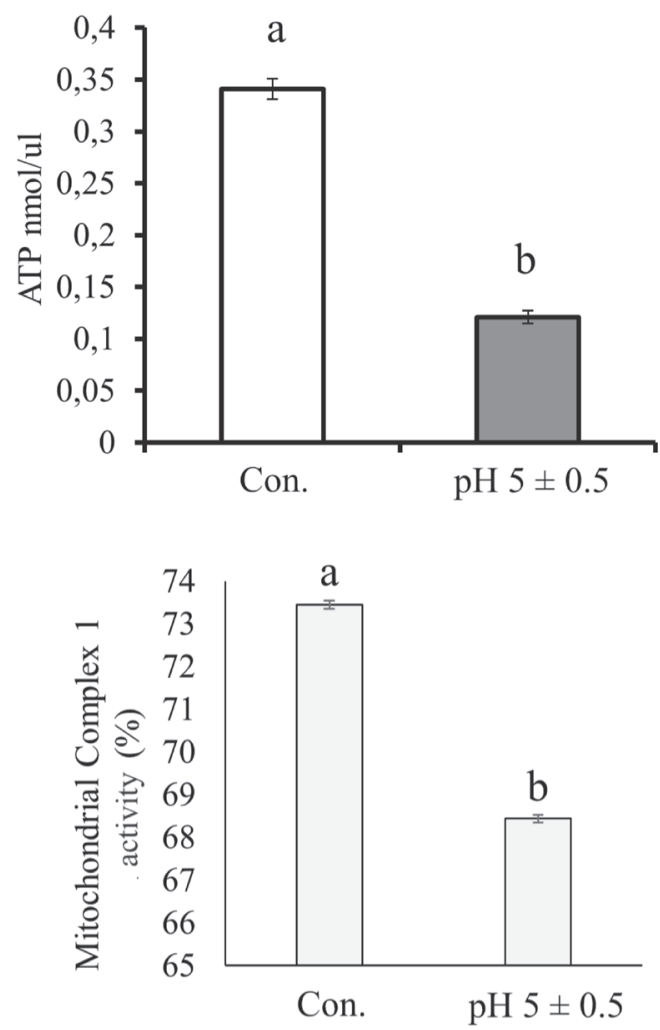

Figure 5. Immunoblots of Tfam, COXII and Nrf1 along with ATP synthesis and mitochondrial complex I activity. (A) Immunoblots of Tfam, COXII and Nrf1 and, their densitometry analysis after $2 \mathrm{~h}$ of stress exposure of zebrafish to $\mathrm{pH} 5.0 \pm 0.5$. (B) ATP production and mitochondrial complex I activity in skeletal muscle tissue on exposure of zebrafish to $\mathrm{pH} 5.0 \pm 0.5$ stress exposure of $2 \mathrm{~h}$ showing significant decrease in ATP production and mitochondrial complex I activity after $2 \mathrm{~h}$. Data are shown as Mean $\pm \mathrm{SE}$. Bars with different lower-case letters show statistically significant difference at $\mathrm{p}<0.05$.

volved in mitochondrial biogenesis) and COXII (one of the essential subunits of the complex IV of electron transport chain). Indeed, significant decrease of $\operatorname{Tfam}\left(\chi^{2}=5.026\right.$, $\mathrm{p}=0.025), \operatorname{Nrf1}\left(\chi_{2}=8.308, \mathrm{p}=0.004\right)$ and COXII $(\chi 2=$ $8.308, \mathrm{p}=0.004)$ protein levels were observed at $\mathrm{pH} 5 \pm$ 0.5 (Fig. 5A). Under stress condition at $\mathrm{pH} 5.0 \pm 0.5$, the production of ATP and mitochondrial complex I activity of electron transport chain also showed a significant reduction $(\chi 2=5.333, \mathrm{p}=0.021$ and $\chi 2=14.329, \mathrm{p}=0.00$, respectively) that may correlate with the damage to the mitochondrial bioenergetics (Fig. 5B).

\section{DISCUSSION}

It is evident from the result that the zebrafish skeletal muscle experienced highest oxidative stress on exposure for $30 \mathrm{~min}$ to $1 \mathrm{~h}$ to acidic ambience. However, the oxidative stress indicators steeply declined from 1-2 h. It is not confirmed whether the physiological health of the fish came under threat for such short exposure or not. The assessment of the formation of MDA is also significantly higher in its skeletal muscle tissue compared to brain and gill under low $\mathrm{pH}$ condition, indicating the resultant post traumatic physiological challenge. Such outcome was also confirmed by the changes in HIF-1 $\alpha$ levels and other stress indicators like SOD and catalase activity and glutathione level at $\mathrm{pH} 5.0 \pm 0.5$ at $2 \mathrm{~h}$. The $15 \%$ mortality at $2 \mathrm{~h}$ further supported the possible failure of the fish to withstand the oxidative stress induced damage. As outlined earlier, the present study aims to understand the metabolic impairment at this point faced by zebrafish through AMPK-SIRT1-PGC1 $\alpha$ axis under acidic ambiance.

Earlier, few studies reported that zebrafish can tolerate pH from 4.0-10.7 (Kwong et al. 2014; Zahangir et al. 2015). However, in the present study, results of oxidative stress indicators confirmed that the skeletal muscle of the fish experienced high oxidative stress within 1 hour to the exposure of $\mathrm{pH} 5.0 \pm 0.5$. The use of MDA and HIF- $1 \alpha$ 
as biomarker of stress further established that the aftereffects of oxidative stress exists even though the oxidative stress indicators were declined in the skeletal muscle at $2 \mathrm{~h}$. Thus, a cellular hypoxic condition might persist even after the exhibition of surge evident from oxidative stress indicators. Such possibility cannot be ignored since the fish displayed unrest behaviour after exposure of 2 $h$ in acidic ambiance with more frequent gulping at the water surface. Although, this level of $\mathrm{pH}$ is not lethal in general, it led the fish to suffer from high oxidative stress, which is reflected from the observation discussed above. Probably, such observation was overlooked by previous researchers, since their studies were not based on formation of MDA in skeletal muscle tissue. In general, MDA is suggested as the efficient stress-marker for animals, especially mammals (Nielsen et al. 1997). Being one of the final products of polyunsaturated fatty acids peroxidation in the cells, its formation indicates the generation of free radicals in tissues (Gawel et al. 2004; Dahake et al. 2016). As mentioned earlier, the results shown by MDA were correlated to the results of stress indicators (SOD, catalase and glutathione). These stress indicators are the part of antioxidant defence system which are either enzymatic (SOD and catalase) or non-enzymatic (glutathione) and are frequently used to evaluate oxidative stress level in animals (Huang et al. 2020). The enhanced level of HIF- $1 \alpha$ protein indicates insufficient $\mathrm{O}_{2}$ in cell that creates hypoxia and mediates the re-establishment of homeostasis in oxidatively stressed cell (Samenza 2005). The cellular hypoxic condition probably persists, even at $2 \mathrm{~h}$, when antioxidant surge exhausted. Under such condition, amount of HIF- $1 \alpha$ increases, as seen in the present study, and helps to generate adaptive response in cell through metabolic and bioenergetic pathways arresting ATP production through decrease in expression of metabolic sensors like AMPK, PGC1 $\alpha$ and SIRT1 (Muoio and Kove 2007; Majumdar et al. 2010). In this study, the increase of HIF-1 $\alpha$ protein level was indeed accompanied by decrease of AMPK, PGC1 $\alpha$ and SIRT1.

Out of all metabolic sensors, the AMPK and SIRT1 act as sensors of cellular energy status for restoration of cellular damage (Han et al. 2013). This homeostatic defence mechanism is a natural biological phenomenon and takes place in all eukaryotes (Hardie et al. 2012a, 2012b; Hardie 2013; Han et al. 2016). The activation of AMPK is found to be linked with increase in AMP:ATP ratio and increased ADP concentrations, both of which are linked to an energetic drop (Canto and Auwerx 2009; Hardie 2011; Mihaylova and Shaw 2011). In case of oxidative stress, the AMPK works as a cellular sensor for degraded AMP:ATP and ADP:ATP ratios and gets activated to recover the energy balance (Auciello et al. 2014). Under the acidic ambiance, in the skeletal muscle of zebrafish, the levels of
AMPK and SIRT1 proteins significantly decreased within $2 \mathrm{~h}$ compared to the stress-free condition, indicating a direct effect of pH $5.0 \pm 0.5$ on skeletal muscle tissue.

It is well known that the master metabolic regulator PGC1 $\alpha$ that promotes the expression of mitochondrial genes, functions synergistically with AMPK and SIRT1 for ATP production under oxidative stress conditions (Canto and Auwerx 2009; Jeninga et al. 2010). In compliance to the above role, the level of PGC1 $\alpha$ protein was found to be significantly decreased in the present study. This directly indicates that the energy synthesis processes are intervened at acidic $\mathrm{pH}$ level of $5.0 \pm 0.5$. The present study also showed significant decrease of mitochondrial complex I activity and COXII protein level that directly indicates a decreased performance of mitochondrial functions. Indeed, reduction in activities of both mitochondrial complex I and COXII, which are parts of the electron transport chain, is indicative of impaired mitochondrial bioenergetics (Rato et al. 2014). Similarly, decrease of AMPK levels results in reduced activation of PGC1 $\alpha$, which in turn results in the perturbation of normal mitochondrial biogenesis processes. The decrease of mitochondrial functions is further reflected by the significantly decreased levels of Tfam and Nrf1 proteins under acidic ambiance compared to control conditions. In mammals, these two transcription factors are directly involved in mitochondrial biogenesis under stress condition (Sharma et al. 2013). There is decrease in mitochondrial derived ATP production in mammals under oxidative stress (Zhang et al. 2006). Congruently, in the present study, the mitochondrial bioenergetics seems to be disrupted resulting in low ATP production under acidic ambiance.

\section{CONCLUSION}

This study explains that zebrafish experiences oxidative stress in skeletal muscle when ambiance turned to acidic, to a level at pH $5.0 \pm 0.5$ within $30 \mathrm{~min}$ to $1 \mathrm{~h}$, which was evident from the response of oxidative stress indicators (SOD and catalase activities and glutathione level). Beyond this point, even though the oxidative stress indicators (SOD and catalase activities and glutathione level) declined, the oxidative stress induced traumatic condition persists. This is evident from the analysis of MDA or HIF1 $\alpha$ levels or other regulators related to energy homeostasis associated directly or indirectly with mitochondria (such as PGC1 $\alpha$ ). The fish, although strived to resume adaptive homeostatic strategy at $\mathrm{pH} 5.0 \pm 0.5$, the increase in the rate of mortality explains state of metabolic dysfunction. Surprisingly, when the fish was kept under acidic ambiance for a period up to $9 \mathrm{~h}$, the rate of mortality reached up to 
90\% and MDA level was reduced to $1000 \mathrm{nmol} \mathrm{MDA} / \mathrm{mg}$ protein. Whether these adaptations at miniscule scale of $10 \%$ is due to naturally inherent counter mechanism or it is just a failure of progressive rate of adaptive response in $90 \%$ of population, is not clear. As of now, this study hints that, acidic ambiance induced post oxidative stress affects the mitochondrial biogenesis and bioenergetics in zebrafish greatly impairing its metabolic adaptability.

\section{ACKNOWLEDGEMENT}

Authors acknowledge National Agriculture Science Fund (NASF) [Project Ref. NASF/ABA/6018/2016-17], The Indian Council of Agricultural Research (ICAR) for financial assistance and DIST FIST- II and CAS-II of Department of Zoology, Visva-Bharati for instrumental assistance. Authors are also thankful to DST-PURSE of Siksha Bhavana, Visva-Bharati for technical assistance.

\section{REFERENCES}

Andrade TS, Henriques JF, Almeida AR, Soares AMVM, Scholz S, Domingues I (2017) Zebrafish embryo tolerance to environmental stress factors-Concentration-dose response analysis of oxygen limitation, $\mathrm{pH}$, and UV-light irradiation. Environ Toxicol Chem 36:682-690.

Auciello FR, Ross FA, Ikematsu N, Hardie DG (2014) Oxidative stress activates AMPK in cultured cells primarily by increasing cellular AMP and/or ADP. FEBS Lett 588:3361-3366.

Aust SD (1985) Lipid peroxidation. In Greenwald RA, ed., Handbook Methods for Oxygen Radical Research. CRC Press Revivals, Florida, 203-210.

Bhaskar M, Govindappa S (1985) Physiological and metabolic patterns in muscle of fish, Tilapia mossambica on acclimation to altered $\mathrm{pH}$. Ambio 14:349-351.

Canto C, Auwerx J (2009) PGC- $1 \alpha$, SIRT1 and AMPK, an energy sensing network that controls energy expenditure. Curr Opin Lipidol 20:98-105.

Dahake HS, Warade J, Kansara GS, Pawade Y, Gangle S (2016) Study of malondialdehyde as an oxidative stress marker in schizophrenia. IJRMS 4:4730-4734.

Dave G (1984) Effect of pH on pentachlorophenol toxicity to embryos and larvae of zebrafish (Brachydanio rerio). Bull Environ Contam Toxicol 33:621-630.

Dave G (1985) The influence of $\mathrm{pH}$ on the toxicity of aluminium, cadmium and iron to eggs and larvae of the zebrafish, Brachydanio rerio. Ecotoxicol Environ Saf 10:253-267.

Ewing JF, Janero DR (1995) Microplate superoxide dismutase assay employing a nonenzymatic superoxide generator.
Anal Biochem 232:243-248.

Eze JI, Anene BM, Chukwu CC (2008) Determination of serum and organ malondialdehyde (MDA) concentration, a lipid peroxidation index, in Trypanosoma brucei infected rat. Comp Clin Pathol 17:67-72

Gawel S, Wardas M, Niedworok E, Wardas P (2004) Malondialdehyde (MDA) as a lipid peroxidation marker. WIAD 57:453-455.

Han G, Zhang S, Marshall DJ, Ke C, Dong Y (2013) Metabolic energy sensors (AMPK and SIRT1), protein carbonylation and cardiac failure as biomarkers of thermal stress in an intertidal limpet: linking energetic allocation with environmental temperature during aerial emersion. J Exp Biol 216:3273-3282.

Han X, Tai H, Wang X, Wang Z, Zhou J, Wei X, Ding Y, Gong H, Mo C, Zhang J, Qin J, Ma Y, Huang N, Xiang R, Xiao H (2016) AMPK activation protects cells from oxidative stress-induced senescence via autophagic flux restoration and intracellular $\mathrm{NAD}(+)$ elevation. Aging cell 15:416-427.

Hardie DG, Ross FA, Hawley SA (2012a) AMP-activated protein kinase: A target for drugs both ancient and modern. Chem and Biol 19:1222-1236.

Hardie DG, Ross FA, Hawley SA (2012b) AMPK: A nutrient and energy sensor that maintains energy homeostasis. Nat Rev Mol Cell Biol 13:251-262.

Hardie DG (2013) AMPK: A target for drugs and natural products with effects on both diabetes and cancer. Diabetes 62:2164-2172.

Hardie DG (2011) Signal transduction: How cells sense energy. Nature 472:176-177.

Huang X, Li Y, Wang T, Liu H, Shi J, Zhang X (2020) Evaluation of the oxidative stress status in zebrafish (Danio rerio) liver induced by three typical organic UV filters (BP-4, PABA, PBSA). Int J Environ Res Public Health 17:1-13.

Jeninga EH, Schoonjans K, Auwerx J (2010) Reversible acetylation of PGC-1: connecting energy sensors and effectors to guarantee metabolic flexibility. Oncogene 29:4617-4624.

Kwong RWM, Kumai Y, Perry SF (2014) The physiology of fish at low $\mathrm{pH}$ : the zebrafish as a model system. J Exp Biol 217:651-662.

Majumdar AJ, Wong WJ, Simon MC (2010) Hypoxia inducible factors and the response to hypoxic stress. Mol Cell 40:294-309.

Mcdonald DG (1983) The effects of H+ upon the gills of freshwater fish. Can J Zool 61:691-703.

Mihaylova MM, Shaw RJ (2011) The AMPK signalling pathway coordinates cell growth, autophagy and metabolism. Nat Cell Biol 13:1016-1023.

Miranda S, Foncea R, Guerrero J, Leighton F (1999) Oxidative stress and upregulation of mitochondrial biogenesis genes in mitochondrial DNA-depleted HeLa cells. Bio- 
chem Biophys Res Com 258:44-49.

Muoio DM, Koves TR (2007) Skeletal muscle adaptation to fatty acid depends on coordinated actions of the PPARs and PGC1 $\alpha$ : implications for metabolic disease. Appl Physiol Nutr Metab 32:874-883.

Nielsen F, Mikkelsen BB, Nielsen JB, Andersen HR, Grandjean P (1997) Plasma malondialdehyde as biomarker for oxidative stress: reference interval and effects of life-style factors. Clin Chem 43:1209-1214.

Psenner R (1994) Environmental impacts on freshwaters: acidification as a global problem. Sci Total Environ 143:53-61.

Rato L, Duarte AI, Tomas GD, Santos MS, Moreira PI, Socorro S, Cavaco JE, Alves MG, Oliveira PF (2014) Pre-diabetes alter testicular PGC1 $\alpha /$ SIRT3 axis modulating mitochondrial bioenergetics and oxidative stress. Biochim Biophys Acta 1837:335-344.

Samenza GL (2004) Hydroxylation of HIF1: Oxygen sensing at the molecular level. Physiology 19:176-182.

Schindler DW (1988) Effects of acid rain on freshwater ecosystems. Science 239:149-157.
Sharma DR, Sunkaria A, Wani WY, Sharma RK, Kandimalla RJL, Bal A, Gill KD (2013) Aluminium induced oxidative stress results in decreased mitochondrial biogenesis via modulation of PGC- $1 \alpha$ expression. Toxicol Appl Pharm 273:365-380.

Steenberger PJ, Richardson MK, Champagne DL (2011) The use of the zebrafish model in stress research. Prog Neuropsychopharmacol Biol Psychiatry 35:1432-1451.

Wood CM (1989) The physiological problems of fish in acid waters. In Morris R, Brown DJA, Taylor EW, Brown JA, ed., Acid Toxicity and Aquatic Animals. Cambridge University Press, England, 125-152.

Zahangir MM, Haque F, Islam MS (2015) Effects of acute water $\mathrm{pH}$ stress on the stress indicators in zebrafish (Danio rerio). Proceedings of 5th International Conference on Environmental Aspects of Bangladesh (ICEAB 2014). Paper ID E76.

Zhang X, Wu XQ, Lu S, Guo YL, Ma X (2006) Deficit of mitochondria-derived ATP during oxidative stress impairs mouse MII oocyte spindles. Cell Res 16:841-850. 